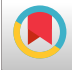

\title{
Burden of Tuberculosis and Challenges Facing Its Eradication in West Africa
}

\author{
Yusuff Adebayo Adebisi ${ }^{1,{ }^{*},}$, Idoko Agumage ${ }^{2}$, Tommy Daniel Sylvanus ${ }^{3}$, Isaac Joshua Nawaila ${ }^{4}$, \\ Williams Alison Ekwere ${ }^{5}$, Mercy Nasiru ${ }^{6}$, Eduoku Emem Okon ${ }^{3}$, Aniekan Michael Ekpenyong ${ }^{3}$ and \\ Don Eliseo Lucero-Prisno III (iD ${ }^{7}$
}

${ }^{1}$ Faculty of Pharmacy, University of Ibadan, Ibadan, Nigeria

${ }^{2}$ Department of Pharmacognosy, University of Lagos, Lagos, Nigeria

${ }^{3}$ Faculty of Pharmacy, University of Uyo, Uyo, Nigeria

${ }^{4}$ Faculty of Pharmacy, Gombe State University, Gombe, Nigeria

${ }^{5}$ Faculty of Pharmaceutical Sciences, University of Port Harcourt, Port Harcourt, Nigeria

${ }^{6}$ Faculty of Pharmaceutical Sciences, University of Jos, Jos, Nigeria

${ }^{7}$ Faculty of Management and Development Studies, University of the Philippines Open University, Los Banos, Philippines

"Corresponding author: Faculty of Pharmacy, University of Ibadan, Ibadan, Nigeria. Email: adebisiyusuff23@yahoo.com

Received 2019 April 14; Revised 2019 September 08; Accepted 2019 September 23.

\begin{abstract}
Context: Tuberculosis (TB) is a major public health challenge, especially in resource-limited settings. The burden of the disease is particularly larger in developing countries due to factors like poverty, undernutrition, and even HIV, which propagate its spread and complicates its control. West Africa, with its high levels of poverty, hunger, overcrowding, and infectious diseases like HIV, is not left out in the tuberculosis epidemic. Our study aimed to review the burden of TB and the challenges hindering its eradication in West Africa.

Evidence Acquisition: This review assesses available evidence on issues relating to the burden of tuberculosis and the challenges facing its eradication in West Africa. Search for relevant medical literature in biomedical databases such as PubMed, Google Scholars, and OVID was conducted with the appropriate key terms without date restriction. Fifty-seven articles were found in a search through the database; 33 data sources, including reports, were selected and reviewed in order to contribute data to this study.

Results: From the study, the incidence of tuberculosis is high in West Africa, especially considering the high rate of factors, which propagate its spread. TB/HIV co-infection is also an issue in this region, as evidenced by the high burdens in Nigeria, Ghana, Liberia, and Guinea Bissau. In fact, Nigeria still holds the position of the country with the highest TB burden in West Africa and also account for about $4 \%$ of the TB incidence globally. However, the burden only represents an estimate due to paucity of data attributed to ineffective surveillance method. The rate at which the incidence of tuberculosis is declining is slow due to various challenges facing its eradication such as poverty, endemic of the causative agents, drug resistant tuberculosis, and inefficient diagnostic methods, among others.

Conclusions: There is now increasing evidence to support that TB prevalence in West Africa is on a continual projection in which the futuristic outcome is worrisome, considering the challenges the region continually faces. The challenges need to be addressed by selecting the most appropriate strategy for the region, and efforts should be made to improve the surveillance system. Leveraging on the public-private partnership and cost-effectiveness evaluation should also be encouraged.
\end{abstract}

Keywords: Tuberculosis, Burden, West Africa, Challenges, Epidemiology, Eradication, HIV, Public Health, Mortality Rate, Drug Resistance

\section{Context}

The major culprit for Tuberculosis (TB) is Mycobacterium tuberculosis, which principally affects the lungs (pulmonary TB) and, less often, other regions of the body (extrapulmonary TB). Tuberculosis is also occasionally caused by other bacilli such as Mycobacterium africanum, which sometimes appears in West Africa and Mycobacterium bo- vis, which causes tuberculosis in domestic or wild cattle but can also cause tuberculosis in humans (1). Not everyone who is infected with the causative bacteria of TB develops the disease. The prevalence of the TB disease is very much greater among HIV seropositive patients (due to weaken immune system), and also higher among people who smoke, are diabetic, undernourished, and drink alcohol excessively (2). Some of the common symptoms

Copyright (c) 2019, International Journal of Infection. This is an open-access article distributed under the terms of the Creative Commons Attribution-NonCommercial 4.0 International License (http://creativecommons.org/licenses/by-nc/4.0/) which permits copy and redistribute the material just in noncommercial usages, provided the original work is properly cited. 
among patients with the TB disease in the lungs include chronic cough, pain in the chest, hemoptysis, or sputum (with phlegm) (3). In addition to these, other noticeable symptoms of TB disease include general body weakness (fatigue), loss of weight (cachexia), anorexia, chills, pyrexia (fever), and nocturnal hyperhidrosis (night sweating) (3).

Although quite curable, TB, in the absence of medical treatment is associated with a high mortality rate. It has been shown that in the absence of treatment, after a period of 1.5 years, approximately a quarter of the patients die, half of those who survive become chronically infected and continue to excrete the bacillus for many years, and the remainder are spontaneously cured by the body's natural defense mechanism (1). The causative agent of TB had been identified for well over a century and its treatment regimens are $100 \%$ effective, however, its impact has been minimal, and this has been as a result of the socio-economic aspect of the disease (4). The effect of TB on the population has been seen to be enormous. It has been observed to have the greatest effect on the productive population of men and women, impacting the economy negatively (2).

$\mathrm{TB}$ poses a major challenge in resource-limited settings, particularly in Africa and some regions of Asia, leading to loss of healthy life years as it leads to a reduction in the global workforce, which in a way have impacts on the economy. TB serves as one of the main causes of deaths related to antimicrobial resistance and the number one killer of people living with HIV, especially in Africa (2). West Africa, home to over 300 million people, is a region in Africa still facing challenges in eradicating TB as evidenced by the high burden imposed by TB, multidrug resistance TB (MDR-TB), and TB/HIV infection. Some of the factors that predispose this region to various infections, including TB are the finite sub-regional health and limited good laboratory footings (5). The incidence of TB on the global scale is declining at the rate of about $2 \%$ per year compared to the $4 \%$ - 5\% needed per annum to arrive at achieving the 2020 target of the end TB strategy (6).

\section{Evidence Acquisition}

This review assesses available evidence on issues relating to the burden of tuberculosis and the challenges facing its eradication in West Africa. The review is qualitativebased. Search for relevant medical literature in biomedical databases such as PubMed, Google Scholars, and OVID was conducted with the following key terms: "Burden", "Epidemiology", "West Africa", "Challenges" and "Tuberculosis". Paper selections were conducted by reviewing their abstracts and titles, in addition, using supplemental references gotten from the reference lists of the papers. In addition to this, data were gotten from WHO 2016 and 2017 tu- berculosis report, CDC report, country report, and World Bank report. Fifty-seven articles were found in a search through the database; 33 data sources, including reports, were selected and reviewed in order to contribute data to this study. No date restrictions on the search for literature were observed.

\section{Epidemiology of Tuberculosis}

In resource-limited regions, TB remains one of the leading global public health challenges (7), which is supported by the declaration made by the World Health Organization (WHO) in 1993, and thus, TB is a global emergency (8). TB is ranked the 9th position of the leading cause of death globally. In addition, it is also the leading cause of death from a single infectious agent within a 5-year period (2012 - 2016), which is even ranked above HIV/AIDS (2). The backbone of TB spread on the global scale includes poverty, HIV, and antimicrobial resistance (9). Based on estimation, just about $5 \%$ to $15 \%$ will develop TB disease in their existence out of the estimated 1.7 billion people infected with M. tuberculosis (2).

In 2016, the estimated incident cases of TB were 10.4 million, with a range of 8.8 million to 12.2 million globally, and it is equivalent to 14 cases per 10000 population (2). Three West African countries (Sierra Leone, Nigeria, and Liberia) made it to top 30 highly epidemiological burdened countries, as reported in the WHO global TB report for 2017 (2). Some of the influencing factors of the TB epidemic include poverty, HIV, undernutrition, and smoking. Of the 10.4 million incident cases of TB in 2016, about 1.9 million were due to poor feeding habit, 1.0 million were caused by immunosuppression due to HIV, 0.8 million were due to smoking, and 0.8 million were attributed to chronic diseases such as diabetes (2). Globally, the number of incident cases in both male and female corresponds to $65 \%$ and $35 \%$, respectively, and $90 \%$ and $10 \%$ of the incident cases correspond to adult and children, respectively (2). Based on studies conducted prior to the availability of drug treatment, it was discovered that in the absence of treatment, within 10 years of being diagnosed up to 7 out of 10 of those patients with sputum smear-positive TB will die, as oppose about two out of 10 of people with culturepositive but who are smear-negative for TB (10). In 2016, treatment success remained as low as 54\% globally (2).

\section{Burden of Tuberculosis in West Africa}

Studies have shown that about 95\% of TB cases occur among the resources limited communities of the developing countries of the world. West Africa, with a population 
of about 376 million people, is not spared as poverty and lack of development keep propagating the spread of TB. In the year 2016, globally, WHO African region accounted for $25 \%$ of the estimated number of TB incident cases. Also, in 2015, the number of estimated TB cases reported in Liberia, Guinea, and Sierra Leone, which are the post-Ebola countries was estimated to be 29809 (2). Nigeria, Sierra Leone, and Liberia are among the list of 30 high encumbered countries for TB. In fact, the incidence of TB in Nigeria in 2016 was estimated to be 219 cases per 100000 population, which account for about $4 \%$ of the TB incidence, globally (2). Liberia and Sierra Leone TB incidence rate was estimated to be 308 and 304 cases per 100000 population; respectively, however, Nigeria still holds the position of the country with the highest TB burden in West Africa with an absolute estimated incidence of 407000 cases in 2016 (2).

Nigeria is also on the list of the 30 high burden countries for TB/HIV with an incidence of 63000 , alongside Ghana, Liberia, and Guinea Bissau (Table 1) (2). An estimate from the WHO global report 2016 revealed that about 93000 cases of MDR-TB occurred in the WHO African region, with Nigeria having 20000 of these cases (2). In 2015 - 2016, the premier national drug resistance investigation was done in Burkina Faso and Ghana and Recurrence probe were completed in Cote d'Ivoire (2). According to subnational data assumptions based on WHO estimate, MDRTB cases in Mali was 130 among notified pulmonary TB patients owing to the fact that no official drug resistance investigation has been conducted until 2016/2017 (11). In 2016 -2017, drug resistance probes were going on in eleven countries, including Mali and Togo, with their first nationwide surveys (2).

At the global level, it has been noted that tuberculosis is accountable for claiming more years of healthy potential life lost much more than other infectious diseases including HIV/AIDS and malaria (12, 13). The 2016 estimated number of deaths recorded were among HIV negative (1.3 million) and HIV-positive (374000) TB infected people (2). Globally, the case fatality ratio (CFR) of TB is about $16 \%$; nonetheless, in the WHO African region, there is a significant country disparity in the CFR, which ranges from less than $5 \%$ in not many countries to greater than $20 \%$ in most countries (2). From the 2017 WHO report, 417000 TB deaths were recorded in the WHO African region among HIV negative people, with 115000 deaths from Nigeria alone, ranking the highest in the region and third globally, after India and Indonesia (2).

The social and economic impacts of tuberculosis are reported frequently, but little or no reports exist on its differential impact on men and women (14). Data received has shown that usually greater than $30 \%$ of the total health expenditures in most high TB burden countries were ac- counted for by out-of-pocket expenditures in a huge proportion (2). In addition, there are incidental costs as a result of lost earnings and productivity that sets in when TB patients are too ill to work, and when their parents and householders die young (15). Outside limitations of these losses, ineptly expressed in the disability-adjusted life years (DALYs) and dollars, enormous cognitive and communal public costs are attributed to TB (16). Although not easily quantified, these extra costs are nonetheless real (16).

\section{Challenges Facing the Eradication of Tuberculosis in West Africa}

Despite the advances in eradicating TB globally, the factors which propagate the development and spread of the disease are numerous in West Africa, impeding the eradication of tuberculosis in this region. Limitation to funding, laboratory capacity, medicine supplies, personnel, and facilities are the leading constraints to the potentiality of the health care system in developing world to reduce or eradicate the emerging TB cases (17). West Africa constitutes a substantial part of the total African population. In addition to a weak health care system, the region faces the HIV epidemic and frequent outbreaks of other infectious diseases, all of which compounds the problem of TB in this region.

\section{Poverty and Undernutrition}

Poverty and undernutrition are big challenges in West Africa fueling the TB epidemic. The escalation of TB cases is closely parallel to the state of poverty as it mainly affects the most liable populations in the world's poorest countries (18). The poor populations are more likely to contract TB due to their often-crowded living conditions. In addition, because of the large population size that is affected, undernutrition caused virtually double as many TB cases as HIV (2). The probability of being infected with TB and becoming symptomatic are associated with household crowding, poor ventilation, malnutrition, and poor sanitation, which are also associated with poverty (15). In the West African countries, persistent poverty and the inequality of rise in income remains one of the socioeconomic problems facing them (19); making this region one of the world's poorest. Fifty percent of the countries that are adjudged to have the world's lowest human development indicators are in West Africa (20). From 2004 through 2010, Nigeria recorded the highest growth rate, yet there was no tangible increase in the poverty reduction rate (19). Environmental and ecological conditions, 


\begin{tabular}{|c|c|c|c|c|}
\hline Countries & Population (Millions) & Incidence $^{\mathbf{b}}$ (Including HIV) & Incidence $^{\mathrm{b}}$ (HIV-Positive) & Incidence $^{\mathrm{b}}$ (MDR/RR-TB) \\
\hline Benin & 11 & $6.4(4.1-9.2)$ & $0.95(0.61-1.4)$ & $0.097(<0.01-0.19)$ \\
\hline Burkina Faso & 19 & $9.4(6.1-13)$ & $0.87(0.55-1.3)$ & $0.3(0.18-0.41)$ \\
\hline Cabo Verde & $<1$ & $0.74(0.48-1.1)$ & $0.06(0.038-0.087)$ & $0.029(<0.01-0.048)$ \\
\hline Côte d'Ivoire & 24 & $36(23-52)$ & $7.9(5.0-11)$ & $2.1(1.1-3.1)$ \\
\hline Gambia & 2 & $3.5(2.7-4.5)$ & $0.65(0.49-0.84)$ & $0.11(0.018-0.20)$ \\
\hline Ghana & 28 & $44(21-75)$ & $10(4.7-17)$ & $0.84(0.27-1.4)$ \\
\hline Guinea & 12 & $22(14-31)$ & $5.4(3.4-7.8)$ & $0.73(0.14-1.3)$ \\
\hline Guinea-Bissau & 2 & $6.8(4.4-9.7)$ & $2.2(1.4-3.2)$ & $0.2(0.017-0.39)$ \\
\hline Liberia & 5 & $14(9.2-20)$ & $2.2(1.4-3.2)$ & $0.43(0.046-0.82)$ \\
\hline Mali & 18 & $10(6.6-14)$ & $1.4(0.90-2.0)$ & $0.38(0.10-0.66)$ \\
\hline Mauritania & 4 & $4.4(2.8-6.3)$ & $0.22(0.084-0.42)$ & $0.14(0.023-0.26)$ \\
\hline Niger & 21 & $19(12-27)$ & $0.95(0.60-1.4)$ & $0.66(0.14-1.2)$ \\
\hline Nigeria & 186 & $407(266-579)$ & $63(40-93)$ & $20(12-29)$ \\
\hline Sao Tome and Principe & $<1$ & $0.2(0.19-0.20)$ & $0.037(0.024-0.053)$ & $0.01(<0.01-0.015)$ \\
\hline Senegal & 15 & $22(15-30)$ & $1.4(0.91-1.9)$ & $0.44(0.28-0.61)$ \\
\hline Sierra Leone & 7 & $22(14-32)$ & $3.1(2.0-4.5)$ & $0.72(0.12-1.3)$ \\
\hline Togo & 8 & $3.5(2.8-4.2)$ & $0.77(0.50-1.1)$ & $0.11(0.023-0.20)$ \\
\hline African region & 1020 & $2590(2310-2900)$ & $764(660-876)$ & $93(81-106)$ \\
\hline Global & 7440 & $10400(8770-12200)$ & $1030(915-1150)$ & $601(541-664)$ \\
\hline
\end{tabular}

${ }^{\text {a }}$ Source: WHO global tuberculosis report 2017

${ }^{\mathrm{b}}$ Numbers in thousands

struggling to avoid hunger, poor housing condition, financial constraints, illiteracy, and inimical psycho-social set of conditions are among the vital determining factors of $\mathrm{TB}$, and at the same time do have a great impact on the tendency of TB ill persons to obtain affordable healthcare services (18). Thus, the economic situation directly impacts TB control. There is also little hesitancy that moderate economic growth and increasing impoverishment are parallel to relaxed headway in health consequences (21). In both cases, treatment is either not received or often irregular.

\section{High Financial Burdens}

TB exerts a high economic and financial burden generally, as seen from an investigational survey of value (in terms of costs) encountered by TB patients and their families completed in seven countries including Ghana (2). The financial cost due to the diagnosis and treatment of the patients are born by the patient themselves and their families (16). As such, TB patients and their household usually face highly debilitating and often lamentable gross costs as a result of the disease. From TB patient cost surveys done, the high financial burden of TB disease results from incidental costs such as income loss and other miscellaneous costs (2). Most countries have put in place social protection schemes such as insurance, cash, and food support among others and provide free TB services to TB infected individuals. In spite of the many free policies and insurance covering TB treatment, TB patients and their households still become subject to substantial medical and non-medical cost as repeated investigations like blood test, $\mathrm{X}$-rays, and even computed tomography (CT) scan are most often requested, more so, the prescription of liver protective and ancillary drugs is not uncommon (22). Also, virtually all West African countries have an out of pocket expenditure greater than $30 \%$ of the total health expenditure, notwithstanding the high poverty levels (2). Those who contract tuberculosis are also more likely to fall into poverty, given the economic impacts of the disease (15). Evidence has also shown that the financial burden faced by TB patients and their household often discourage treatment adherence (22). Social protection policies need to be strengthened generally in all West African countries to remove the barrier imposed by the economic burden of TB. 


\section{Burden of HIV}

Perhaps, the greatest challenge to the eradication of tuberculosis in West Africa is the burden of HIV/AIDS. HIV is a major predisposing factor and serves as an opportunistic infection for TB (7). Data gotten from surveillance show that West African countries have accomplished intensive HIV epidemics with upraised HIV pervasiveness among female prostitutes and gays $(23,24)$. However, most West African countries have previously channeled their HIV/AIDS national response to an outbreak on the entire population, with insignificant regards to the key populations. The consequence of this is that there is limited data needful to inform a vast public health response (23). In West African countries with rapid HIV prevalence, more than $75 \%$ of TB cases are HIV-associated (7), with $31 \%$ of the total new cases of TB in adults (aged 15 - 49 years) coinfected with HIV (25). Even though there is growing evidence showing significant HIV burden amid most-at-risk and vulnerable populations, moral debate still remains the leading influencer of the public health response in most West African countries (23). In most of the countries, there is a nonchalant attitude of the government towards engaging with key population associates in expansion and sustainability of programs even when funded nongovernmental or community-based organizations deaden the important programs (23). As such, the HIV/AIDS epidemic facing this region cannot be effectively controlled, and the risk of contracting TB and/or developing the clinical disease remains high (2).

\section{Late Diagnosis and Insensitive Technique of TB De- tection}

Early diagnosis of TB facilitates prompt treatment, limiting the spread of $\mathrm{TB}$, and reducing mortality. However, many TB patients are not promptly diagnosed; instead, they remain ill and contagious for a long period of time before the detection of the cause while many were not even diagnosed before they died (17). Diagnosis is usually at the advanced stages of the TB disease when complications must have set in which is usually due to patient's remedy-seeking behavior, ill-suited diagnostic investigation requested by a clinician, and unsatisfactory capacities for error-free diagnosis at the available health care facilities (26-28). In Africa, the diagnosis of TB depends mainly on microscopically detecting culprit acidfast bacilli (with inconsistent gram stain color) in the patient's sputum, which is an insensitive technique especially among HIV seropositive individuals, who have not many bacilli in their sputum and are prone to more complications such as extra pulmonary tuberculosis as com- pared to HIV seronegative patients (17). In some HIV/TB cases, normal chest radiographs and even negative sputum smears have been observed, resulting in a negative diagnosis in which the exception presence of prominent clinical signs and symptoms is an exception (16). Globally, the use of smear microscopy with the increasing use of a rapid molecular test for diagnostic purposes has been discontinued by many countries. Even though there are advancements in the diagnosis of $\mathrm{TB}$, a reasonable number of TB cases reported to WHO are diagnosed in the clinics and hospitals via physical examinations rather than being confirmed bacteriologically. For instance, the number of cases confirmed via bacteriological procedures reported to WHO in 2016 accounted for only 57\% of the total cases reported (2).

\section{Endemic of the Causative Agents}

Research has shown that the two M. africanum lineages are endemic only in West Africa in the world. In fact, besides the common M. tuberculosis lineage, there are numerous strains of the $M$. africanum lineages which are responsible for up to $40 \%$ of TB infections in this region. The worldwide phylogeographical dispersal of the Mycobacterium tuberculosis complex (MTBc) suggests strain diversity to be pronounced in West Africa with the presence of numerous MTBc pedigrees that are of huge interest (29). Most rapid tests to confirm cultures of TB bacteria are optimized to detect Mycobacterium tuberculosis, which is responsible for most cases worldwide (30). However, infections with Mycobacterium africanum may give a false negative result (more than $20 \%$ of cases) when diagnosed using rapid tests (30). This will fuel an increase in the risk of transmission due to the failure in identification of the MTBc, which will slow down the initiation of necessary treatment. However, the rapid tests still remain the best choice because of its limited costs, the ease of use and the speed with which the results are obtained (30).

\section{Drug-Resistant Tuberculosis and Poor Access to Re- liable Diagnostic Tests}

Likewise, the increasing cases of drug-resistant tuberculosis and unreliable and poor access to diagnostic tests that allow confirming and identifying isolates that are not fully susceptible to antimicrobial first-line drugs further complicates the challenges. MDR-TB is a condition whereby the patient is resistance to not less than one of the first-line antitubercular drugs that is isonicotinohydrazide (isoniazid) and rifampicin while MDR-TB with additional resistance to, at least, any fluoroquinolone 
such as ciprofloxacin and any one of three second-line injectable aminoglycosides (capreomycin, kanamycin, and amikacin) is referred to as extensively drug-resistant TB (XDR-TB) (18). Researches which were carried out in Senegal, Nigeria, Mali, Ghana, Togo, Gambia, Burkina Faso, and Guinea-Bissau by the West African Centre for Excellence for TB, AIDS and Malaria (WANETAM) on TB patients showed that about $39 \%$ of them were resistant to at least a firstline antibiotic used for TB treatment. Based on WHO recommendations, all speculative TB patients are to come in for drug-susceptibility test; however, the implementation is still far behind in many countries due to lack of wellequipped laboratory (13). Therefore, the wrong diagnosis of drug resistance TB may be common in West African countries (31). In-depth studies are imperative to know the true extent of drug resistance in the region. For example, Liberia has never conducted a drug resistance survey even though the country is among one of the top 30 countries with prime TB burden while the resistance survey data for Sierra Leone are those gotten year before 2000 (2).

\section{Ineffective Surveillance System}

For TB control to be carried out, the impact of an effective surveillance system cannot be overemphasized due to the need to define the pattern of the epidemic. In addition to that, assessment of the effectiveness and the overall impacts of control strategies cannot also be deemphasized (18). From the WHO 2017 TB report, majority of the West African countries make use of the opinion of the experts in combination with data gotten from the case notification about case-detection gaps to estimate their TB incidence in the country. These methods are the least preferred and less efficient means by which TB incidence may be estimated and are only relied upon when other methods cannot be used (2). One of the impacts of poor surveillance method is the resulting data gap. For instance, in 2008, only about $14022 \mathrm{~TB}$ cases were reported in Ghana to health facilities which represent about one-third of the actual estimated number of cases (32). Furthermore, despite the prevalence survey method used in Nigeria, on the global scale, the country still accounts for $8 \%$ of the sum gap between cases that are reported and TB incidence (2). When TB cases are not promptly identified and treated, TB control is impeded, and transmission is further increased. Therefore, the country national TB programs (NTPs) and wellmeaning funding agencies need to increase their commitments in conducting and funding TB inventory studies to strengthen the national surveillance systems in West Africa (2).

In all, from the prevalence to notification ratios from surveys implemented in 2007-2016, the rate at which men are accessing available diagnostic and treatment services compared to women are much lower (2). A common factor that discourages TB patients from seeking medical care and adhering to therapy is the fear of stigmatization (18). In addition to this, traditional believes further delay and even prevent TB patient from accessing health care services and getting access to treatment, further fueling TB transmission. Due to the higher burden of disease, coupled with the larger detection and reporting gap in men, there is a need to implement strategies that will improve access to healthcare among men (2). Particularly, more emphasis needs to be placed on clinical trials that will investigate the local health needs in West Africa in order to successfully understand and eradicate the TB epidemic in this region (33).

\section{Results}

This review provided comprehensive data on the burden of TB in West Africa, which will serve as a guide for policymakers, funding bodies, and other stakeholders to enhance immediate intervention. Despite the efforts invested in combating $\mathrm{TB}$ and the fact that most cases of TB can be cured, it still remains a menace in West Africa. Therefore, there is a need to double efforts by stakeholders to enhance the achievement of total eradication of tuberculosis in West Africa. Leveraging on the public-private partnership is another strategy that can be used to reduce financial constraints, which will contribute to a reduction in out-of-pocket expenditure among the key population living below the poverty line and direct investment in more sustainable research for newer drugs, and convenient regimen remain pertinent.

\section{Conclusions}

Tuberculosis still remains one of the major public health problems, especially among HIV patients as an opportunistic disease. A paucity of data, due to an ineffective surveillance method, HIV epidemic, and poverty, still remains major challenges facing the eradication of $\mathrm{TB}$ in West Africa. Others include stigmatization, financial burden, traditional believes, and poor TB diagnostic methods. Although preventive measures against the disease are expanding, most people eligible for preventive therapy are not taking due advantage to access healthcare. The incidence changes continually in West Africa and the rate of declination is slow, as it should be at $4 \%-5 \%$ to reach the first milestone of the end TB strategy. 


\section{Footnotes}

Authors' Contribution: All the authors participated in the search for literature, articles interpretation, analysis of data, and conceptualization. In addition, we all partook in reviewing the final manuscript, and it was read and approved before submission.

Conflict of Interests: The authors declare no competing interests.

\section{Funding/Support: None.}

\section{References}

1. Cosivi O, Grange JM, Daborn CJ, Raviglione MC, Fujikura T, Cousins $\mathrm{D}$, et al. Zoonotic tuberculosis due to Mycobacterium bovis in developing countries. Emerg Infect Dis. 1998;4(1):59-70. doi: 10.3201/eid0401.980108. [PubMed: 9452399]. [PubMed Central: PMC2627667].

2. World Health Organization. Global tuberculosis report 2017. Washington, DC: WHO Global TB Programme; 2017.

3. El-Hazmi MM, Al-Otaibi FE. Predictors of pulmonary involvement in patients with extra-pulmonary tuberculosis. J Family Community Med. 2012;19(2):88-92. doi: 10.4103/2230-8229.98287. [PubMed: 22870411]. [PubMed Central: PMC3410185].

4. Murray CJ. Social, economic and operational research on tuberculosis: Recent studies and some priority questions. Bull Int Union Tuberc Lung Dis. 1991;66(4):149-56. [PubMed: 1687507].

5. Gehre F, Otu J, Kendall L, Forson A, Kwara A, Kudzawu S, et al. The emerging threat of pre-extensively drug-resistant tuberculosis in West Africa: Preparing for large-scale tuberculosis research and drug resistance surveillance. BMC Med. 2016;14(1):160. doi: 10.1186/s12916016-0704-5. [PubMed: 27806714]. [PubMed Central: PMC5094099].

6. World Health Organisation. Tuberculosis: Fact sheets. WHO Media centre; 2017, [August 15, 2019]. Available from: http://www.who.int/ mediacentre/factsheets/fs104/en/.

7. Corbett EL, Bandason T, Cheung YB, Munyati S, Godfrey-Faussett P, Hayes R, et al. Epidemiology of tuberculosis in a high HIV prevalence population provided with enhanced diagnosis of symptomatic disease. PLoS Med. 2007;4(1). e22. doi: 10.1371/journal.pmed.0040022. [PubMed: 17199408]. [PubMed Central: PMC1761052].

8. Zumla A, Abubakar I, Raviglione M, Hoelscher M, Ditiu L, McHugh TD, et al. Drug-resistant tuberculosis-current dilemmas, unanswered questions, challenges, and priority needs. J Infect Dis. 2012;205 Suppl 2:S228-40. doi: 10.1093/infdis/jir858. [PubMed: 22476720].

9. Corbett EL, Marston B, Churchyard GJ, De Cock KM. Tuberculosis in sub-Saharan Africa: Opportunities, challenges, and change in the era of antiretroviral treatment. Lancet. 2006;367(9514):926-37. doi: 10.1016/S0140-6736(06)68383-9. [PubMed: 16546541].

10. Tiemersma EW, van der Werf MJ, Borgdorff MW, Williams BG, Nagelkerke NJ. Natural history of tuberculosis: Duration and fatality of untreated pulmonary tuberculosis in HIV negative patients: A systematic review. PLoS One. 2011;6(4). e17601. doi: 10.1371/journal.pone.0017601. [PubMed: 21483732]. [PubMed Central: PMC3070694].

11. Diarra B, Goita D, Tounkara S, Sanogo M, Baya B, Togo AC, et al. Tuberculosis drug resistance in Bamako, Mali, from 2006 to 2014. BMC Infect Dis. 2016;16(1):714. doi:10.1186/s12879-016-2060-7. [PubMed: 27894266]. [PubMed Central: PMC5126865].

12. World Health Organisation. Child health and development. Department of child and adolescent health; 2002.

13. World Health Organization. Global tuberculosis report2016. WHO;2016.
14. Rajeswari R, Balasubramanian R, Muniyandi M, Geetharamani S, Thresa X, Venkatesan P. Socio-economic impact of tuberculosis on patients and family in India. Int J Tuberc Lung Dis. 1999;3(10):869-77. [PubMed: 10524583].

15. Laokri S, Dramaix-Wilmet M, Kassa F, Anagonou S, Dujardin B. Assessing the economic burden of illness for tuberculosis patients in Benin: Determinants and consequences of catastrophic health expenditures and inequities. Trop Med Int Health. 2014;19(10):1249-58. doi: 10.1111/tmi.12365. [PubMed: 25040399].

16. Peabody JW, Shimkhada R, Tan CJ, Luck J. The burden of disease, economic costs and clinical consequences of tuberculosis in the Philippines. Health Policy Plan. 2005;20(6):347-53. doi: 10.1093/heapol/czi041. [PubMed: 16155066].

17. Chaisson RE, Martinson NA. Tuberculosis in Africa-combating an HIV-driven crisis. N Engl J Med. 2008;358(11):1089-92. doi: 10.1056/NEJMp0800809. [PubMed: 18337598].

18. Sulis G, Roggi A, Matteelli A, Raviglione MC. Tuberculosis: Epidemiology and control. Mediterr J Hematol Infect Dis. 2014;6(1). e2014070. doi: 10.4084/MJHID.2014.070. [PubMed: 25408856]. [PubMed Central: PMC4235436].

19. Dursun G, Ogunleye B. Economic growth, employment and poverty reduction: The case of West African countries. Am JEcon. 2016;6(1):5060.

20. Olofin OP. Foreign aid and poverty level in West African countries: New evidence using a heterogeneous panel analysis. Australian J Bus Manage Res. 2013;3(4):9.

21. Baingana FK, Bos ER. Changing patterns of disease and mortality in Sub-Saharan Africa: An overview. In: Jamison DT, Feachem RG, Makgoba MW, Bos ER, Baingana FK, et al., editors. Disease and mortality in Sub-Saharan Africa. Washington (DC); 2006.

22. Chen S, Zhang H, Pan Y, Long Q, Xiang L, Yao L, et al. Are free antituberculosis drugs enough? An empirical study from three cities in China. Infect Dis Poverty. 2015;4:47. doi: 10.1186/s40249-015-0080-y. [PubMed: 26510711]. [PubMed Central: PMC4625923].

23. Djomand G, Quaye S, Sullivan PS. HIV epidemic among key populations in West Africa. Curr Opin HIV AIDS. 2014;9(5):506-13. doi: 10.1097/COH.0000000000000090. [PubMed: 25010898]. [PubMed Central: PMC4804351].

24. Alary M, Lowndes CM, Van de Perre P, Behanzin L, Batona G, Guedou FA, et al. Scale-up of combination prevention and antiretroviral therapy for female sex workers in West Africa: Time for action. AIDS. 2013;27(9):1369-74. doi: 10.1097/QAD.0b013e32835fd7bd. [PubMed: 23945501].

25. Corbett EL, Watt CJ, Walker N, Maher D, Williams BG, Raviglione MC, et al. The growing burden of tuberculosis: Global trends and interactions with the HIV epidemic. Arch Intern Med.2003;163(9):1009-21. doi: 10.1001/archinte.163.9.1009. [PubMed: 12742798].

26. Bojovic O, Medenica M, Zivkovic D, Rakocevic B, Trajkovic G, Kisic-Tepavcevic D, et al. Factors associated with patient and health system delays in diagnosis and treatment of tuberculosis in Montenegro, 2015-2016. PLoS One. 2018;13(3). e0193997. doi: 10.1371/journal.pone.0193997. [PubMed: 29522545]. [PubMed Central: PMC5844538].

27. Senkoro M, Hinderaker SG, Mfinanga SG, Range N, Kamara DV, Egwaga $S$, et al. Health care-seeking behaviour among people with cough in Tanzania: Findings from a tuberculosis prevalence survey. Int $J \mathrm{Tu}$ berc Lung Dis. 2015;19(6):640-6. doi: 10.5588/ijtld.14.0499. [PubMed: 25946352].

28. Said K, Hella J, Mhalu G, Chiryankubi M, Masika E, Maroa T, et al. Diagnostic delay and associated factors among patients with pulmonary tuberculosis in Dar es Salaam, Tanzania. Infect Dis Poverty. 2017;6(1):64. doi: 10.1186/s40249-017-0276-4. [PubMed: 28335816]. [PubMed Central: PMC5364704].

29. Gagneux S, DeRiemer K, Van T, Kato-Maeda M, de Jong BC, Narayanan $S$, et al. Variable host-pathogen compatibility in Mycobacterium tuberculosis. Proc Natl Acad Sci U S A. 2006;103(8):2869-73. doi: 
10.1073/pnas.0511240103. [PubMed: 16477032]. [PubMed Central: PMC1413851].

30. Ofori-Anyinam B, Kanuteh F, Agbla SC, Adetifa I, Okoi C, Dolganov G, et al. Impact of the Mycobaterium africanum West Africa 2 lineage on TB diagnostics in West Africa: Decreased sensitivity of rapid identification tests in the Gambia. PLoS Negl Trop Dis. 2016;10(7). e0004801. doi: 10.1371/journal.pntd.0004801. [PubMed: 27387550]. [PubMed Central: PMC4936735].

31. Onyedum CC, Alobu I, Ukwaja KN. Prevalence of drug-resistant tuberculosis in Nigeria: A systematic review and meta-analysis. PLoS One. 2017;12(7). e0180996. doi: 10.1371/journal.pone.0180996. [PubMed:
28704459]. [PubMed Central: PMC5509256].

32. Addo KK, Yeboah-Manu D, Dan-Dzide M, Owusu-Darko K, Caulley P, Mensah GI, et al. Diagnosis of tuberculosis in Ghana: The role of laboratory training. Ghana Med J. 2010;44(1):31-6. [PubMed: 21326989]. [PubMed Central: PMC2956310].

33. Gehre F, Kumar S, Kendall L, Ejo M, Secka O, Ofori-Anyinam B, et al. A Mycobacterial perspective on tuberculosis in West Africa: Significant geographical variation of $\mathrm{M}$. africanum and Other $\mathrm{M}$. tuberculosis complex lineages. PLoS Negl Trop Dis. 2016;10(3). e0004408. doi 10.1371/journal.pntd.0004408. [PubMed: 26964059]. [PubMed Central: PMC4786107]. 